\title{
Apoptosis and autophagy alterations are involved in the development of Alzheimer's disease-like pathology in OXYS rats
}

\author{
G.K. Suvorov*, D.V. Telegina, N.A. Stefanova \\ Institute of Cytology and Genetics SB RAS, Novosibirsk, Russia \\ *e-mail: telegina@bionet.nsc.ru
}

Key words: apoptosis, autophagy, Alzheimer's disease, OXYS rats, RNA-seq

Motivation and Aim: Alzheimer's disease (AD) is the most common neurodegenerative disorder and is a cause of dementia that is characterized by accumulation of neurotoxic forms of the peptide amyloid- $\beta(A \beta)$, a hyperphosphorylation of the tau protein, a deficit of synapses, inflammation, mitochondrial dysfunction, oxidative stress, and neuronal death. Autophagy and apoptosis are basic physiologic processes contributing to the maintenance of cellular homeostasis. The proper function and balance in the action of these two type of cell death are especially important in neurons and other long-lived cells. Hence, their dysfunction contributes to pathogeneses of neurodegenerative diseases such as AD but the information on their alterations during development of AD is very limited. Our aim is to researcher the dynamics of apoptosis and autophagy in prefrontal cortex of senescence-accelerated OXYS rats that simulate key aspects of sporadic AD. We investigated three stages of the disease (pre-symptomatic, 20 days; symptomatic, 5 month; and progressive stage, 18 month) in OXYS rats, using RNA-Seq technique and immunohistochemical analysis.

Results: Our results show that the development of the signs of AD (between ages 20 days and 5 months) in OXYS rats takes place during changes in mRNA expression of the 7 genes that are mostly related to processes of autophagy, such as Atg12, Atg7, and Atg8 (regulators of elongation). In addition, changes in mRNA expression of the 21 genes were related to apoptosis (proapoptotic genes and inhibitors) in the prefrontal cortex of OXYS rats between ages 20 days and 5 months. In OXYS rats, with progression of disease, 24 genes related to apoptosis and 7 genes related to autophagy change their expression. Importantly, Wistar rats show changes in expression of 21 genes related to apoptosis only between ages 20 days and 5 months. We also indicated the upregulation of 5 proapoptotic genes in 20-day-old OXYS rats compared Wistar rats. At the age of 5 and 18 months in OXYS rats, the balance between pro- and anti-apoptotic genes were changed (compared to Wistar rats). Using TUNEL kit, we demonstrated that level of apoptosis was increasing in OXYS rats compare age-matched Wistar rats (control) at the age of 20 days, 5 and 18 mo. The analysis of the results of immunohistochemical staining of the brain sections showed that the LC3 A/B (marker of autophagy) was predominant in the glial cells in prefrontal cortex of 20 day-old and 5 mo rats of both lines. The content of LC3 protein in the prefrontal cortex of OXYS and Wistar rats increased by the age of 18 mo and LC3 localized predominantly in neuron. In addition, the extensive colocalization was observed between Mfn1-labeled mitochondria and LC3 A/B at the age of $18 \mathrm{mo}$ in both rats lines that indicated the increasing mitophagy with age.

Conclusion: Accordingly, we demonstrated that the development of AD-like pathology in OXYS rats is related to the alterations in processes of autophagy and apoptosis.

Acknowledgements: Supported by the Russian Science Foundation (project No. 161510005). 